\title{
Epidural catheter positioning in labour: is the transverse echographic approach accurate and precise for the definition of the epidural space?
}

\author{
Perna Paolo1, Gioia Antonio², Ragazzi Riccardo², Innamorato Massimo ${ }^{1}$ \\ 'Ospedale Santa Maria delle Croci, Dept of Anaesthesiology \& Pain Medicine, Ravenna, Italy, \\ ${ }^{2}$ Arcispedale Sant'Anna, Dept of Anaesthesiology \& Intensive Care, Cona, Italy
}
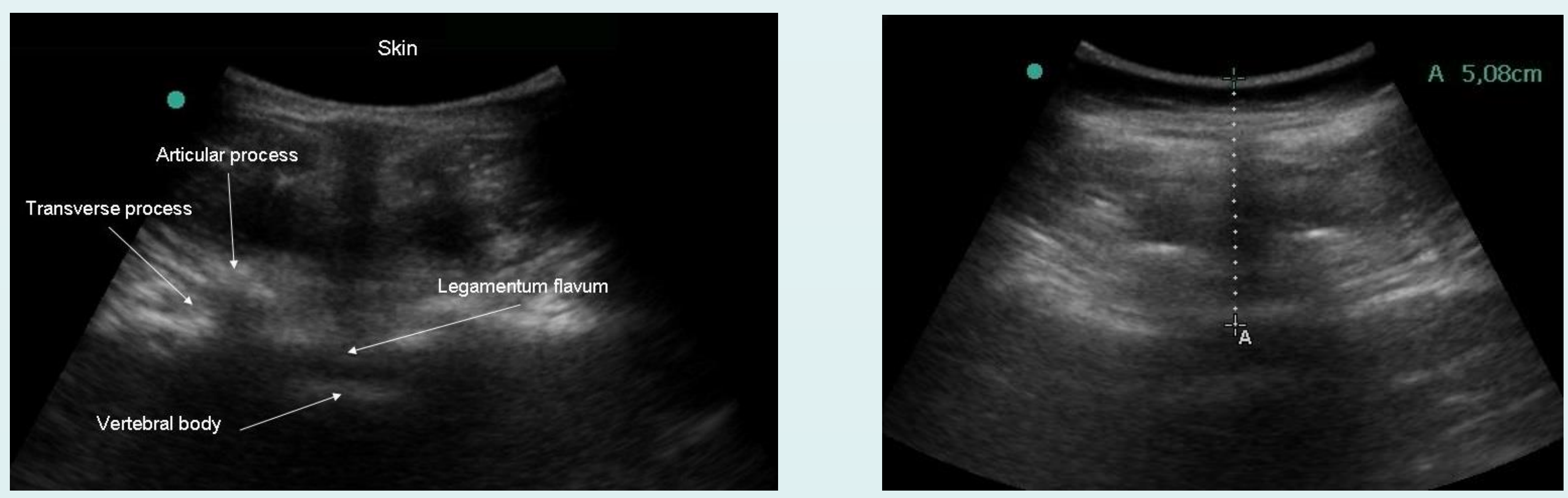

Background and Goal of Study:

The loss of resistance (LOR) technique is the most used for the positioning of the epidural catheter in pregnant women but the anatomic landmarks are not always clear. [1]

The aim of our study was to verify the accuracy and precision of the transverse echographic approach for the definition of the depth of the epidural space.

\section{Materials and methods:}

Prospectic observational study conducted in the Hospital "S. Maria delle Croci" in Ravenna from February to June 2014. The epidural set used was B. Braun Perifix ${ }^{\circledR}$ Filter Soft Tip set. US imaging was performed using a portable SonoSite NanoMax ${ }^{\circledR}$ equipped with a 52 $\mathrm{MHz}$ C60n Convex probe.

The probe was positioned horizontally, perpendicular to the long axis of the spine. In this position, spinous processes correspond to a hyperechoic signal, immediately under the skin, in continuity with a triangular hypoechoic acoustic shadow. The probe was then slightly moved caudally or cephally to define the intervertebral space. Within the interspace, a hyperechoic band was represented on the midline; it corresponded to ligamentum flavum and dorsal dura. A second hyperechoic band, parallel to the first one, represented the anterior dura, the posterior longitudinal ligament and the vertebral body.

By freezing the image, it was possible to measure the ecographic distance between the skin and the first hyperechoic line (distance between skin and posterior dura, also named ultrasound-depth of epidural space, UD).

The distance between the skin and the first hyperechoic line (posterior dura) was named "ultrasound depth", UD.

Tuohy needle was then introduced with LOR technique and a mark was made at the skin level. The distance from the needle tip to the mark was as "real depth" (RD).

Data regarding the whole procedure of epidural analgesia, RD, UD were collected.

\section{References:}

1. Borges B, et al. Reg Anesth Pain Med.2009; 34:581585.

2. Shaikh F, et al. BMJ. 2013; 346:f1720.

3. Arzola C, et al. Anesth Analg. 2007; 104:118892.

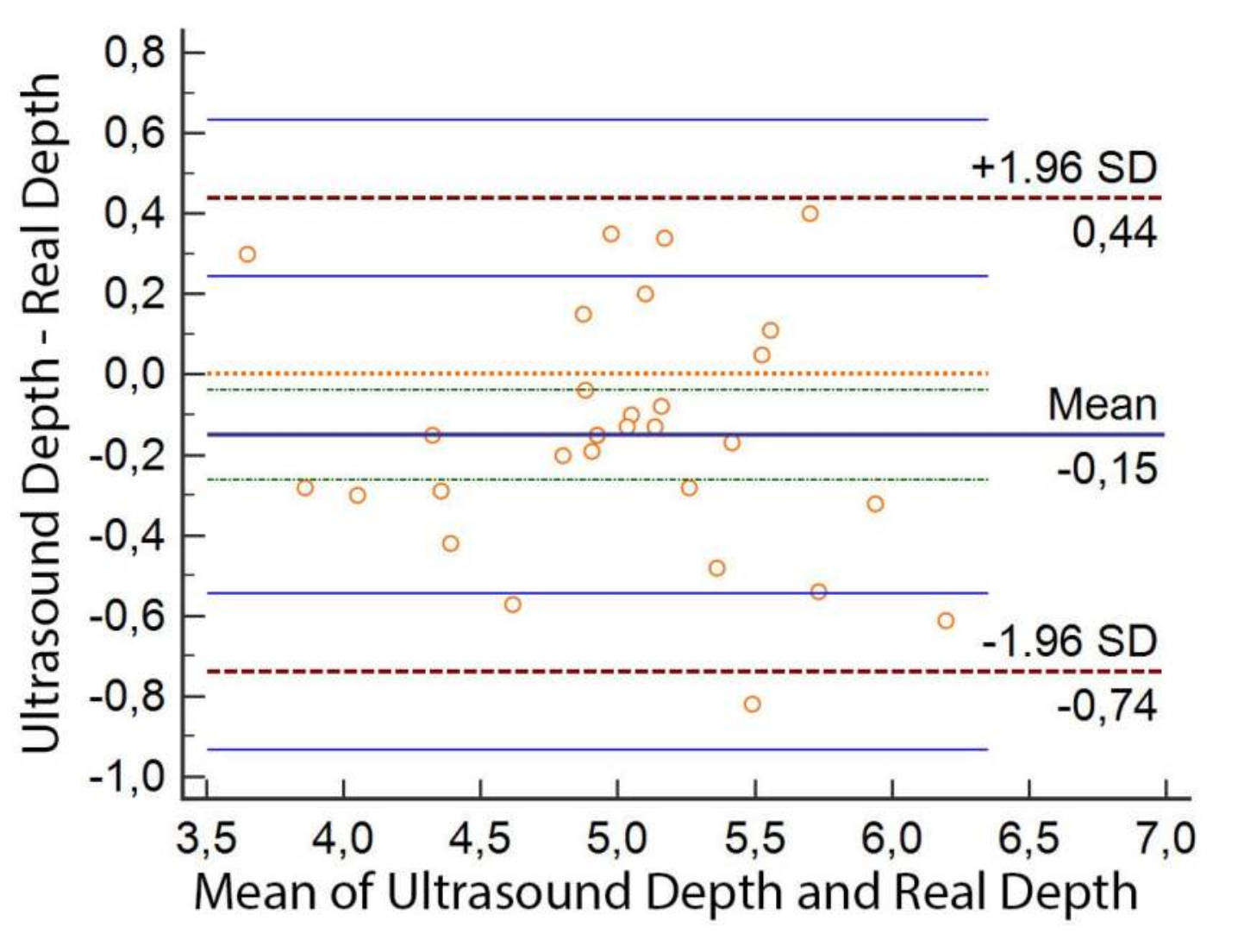

\section{Conclusion:}

US assisted epidural catheter positioning shows a great degree of correlation between UD and RD, but US measure tends to underestimate the RD of the epidural space.

Possible explanations for this discrepancy can be the different trajectory assumed by the needle with respect to the direction of the beam of ultrasounds, or the different tissue compression exerted by the probe during the scan with respect to that of the Tuohy needle during the maneuver. 